\title{
Cooperation between ETS variant 2 and Jumonji domain-containing 2 histone demethylases
}

\author{
XIAOMENG LI ${ }^{1,2}$, GENE MOON ${ }^{2}$, SOOK SHIN ${ }^{2,3}$, BIN ZHANG $^{1}$ and RALF JANKNECHT ${ }^{2,3}$ \\ ${ }^{1}$ China-Japan Union Hospital of Jilin University, Changchun, Jilin 130033, P.R. China; ${ }^{2}$ Department of Cell Biology, \\ University of Oklahoma Health Sciences Center; ${ }^{3}$ Stephenson Cancer Center, Oklahoma, OK 73104, USA
}

Received June 2, 2017; Accepted January 3, 2018

DOI: $10.3892 / \mathrm{mmr} .2018 .8507$

\begin{abstract}
The E26 transformation-specific (ETS) variant 2 (ETV2) protein, also designated as ETS-related 71, is a member of the ETS transcription factor family and is essential for blood and vascular development in the embryo. The role of ETV2 in cancer has not yet been investigated. In the present study, the expression of ETV2 mRNA was identified in a variety of tumor types, including prostate carcinoma. In addition, ETV2 gene amplification was identified in several types of cancer, suggesting that ETV2 plays an oncogenic role in tumorigenesis. It was demonstrated that ETV2 forms complexes with two histone demethylases: Jumonji domain-containing (JMJD)2A and JMJD2D; JMJD2A has been previously reported as a driver of prostate cancer development. In the present study, it was reported that ETV2 exhibited the potential to stimulate the promoters of matrix metalloproteinases (MMPs), including MMP1 and MMP7, within LNCaP prostate cancer cells. JMJD2A and JMJD2D could synergize with ETV2 to activate the MMP1 promoter, whereas only JMJD2A stimulated the MMP7 promoter in cooperation with ETV2. Furthermore, ETV2 expression was positively associated with JMJD2A and JMJD2D mRNA levels in neuroendocrine prostate tumors, in which an ETV2 gene amplification rate of $17.8 \%$ was identified. Collectively, the results of the present study indicated that ETV2, JMJD2A and JMJD2D may jointly promote tumorigenesis, particularly neuroendocrine prostate tumors. In addition, the interaction with the JMJD2A and JMJD2D epigenetic regulators may be important in the ability of ETV2 to reprogram cells, modulate normal and cancer stem cells, and affect spermatogenesis.
\end{abstract}

Correspondence to: Dr Ralf Janknecht, Department of Cell Biology, University of Oklahoma Health Sciences Center, 975 NE 10th Street, BRC-1464, Oklahoma, OK 73104, USA

E-mail: ralf-janknecht@ouhsc.edu

Key words: cancer, ETS-related 71, ETS variant 2, histone demethylase, Jumonji domain-containing 2, lysine demethylase 4, transcription

\section{Introduction}

The ETS variant 2 (ETV2) protein, also designated as ETS-related 71 (ER71), is a DNA-binding transcription factor (1,2); ETV2 belongs to the E26 transformation-specific (ETS) family of proteins, which are characterized by a $\sim 85$ amino acid-long ETS DNA-binding domain and comprises 28 members in humans (3). The knockout of ETV2 in mice has been reported to cause embryonal lethality around day E9.5, indicating that ETV2 is essential during early developmental stages; in particular, ETV2 is required for the development of blood and vascular structures (4,5). Mechanistically, ETV2 facilitates the upregulation of fetal liver kinase (FLK1), a trans-membrane tyrosine kinase and receptor for vascular endothelial growth factor that is crucial for the aforementioned developmental processes. Notably, a lack of FLK1 expression phenocopied ETV2 deficiency in mice (6), emphasizing the biological relevance of the ETV2-FLK1 axis in embryonal blood and vessel formation.

In adult mice, ETV2 expression was reported to be undetectable in endothelial cells and the conditional knockout of ETV2 in the endothelial compartment did not affect adult vasculature or viability. Upon injury, however, ETV2 expression was rapidly upregulated in endothelial cells, and vascular regeneration was dependent on ETV2 in several models, including the hindlimb ischemic injury paradigm. In addition, the delivery of lentivirus-expressing ETV2 into ischemic hindlimbs demonstrated positive therapeutic effects by increasing capillary formation and reducing tissue fibrosis (7). Furthermore, the conditional deletion of ETV2 in adult hematopoietic cells did not affect the viability of mice, but led to a decrease in the number of hematopoietic stem cells. This may not be relevant under normal conditions; however, the stem cell repopulation potential appeared to be adversely affected following damage (8). Thus ETV2 is absolutely critical in hematopoietic and endothelial cells during embryogenesis, and is not essential in these respective adult cells, but may facilitate their response to injury.

Northern blot analyses have revealed elevated ETV2 expression levels in adult mouse testes, whereas other tissues did not exhibit significant levels of ETV2 mRNA expression (1,9). In addition, the ETV2 gene has been identified as a target of the sex-determining region $\mathrm{Y}(\mathrm{SRY})$ protein that is encoded on the $\mathrm{Y}$ chromosome. It has been reported that ETV2 expression 
becomes upregulated within male mouse gonads following the transient expression of SRY during embryogenesis (10), indicating that ETV2 may be involved in the differentiation of the initially bipotential gonads into testes. Furthermore, ETV2 can bind to and activate the promoter of SOX9 $(10,11)$, a transcription factor downstream of SRY. SOX9 is important for male differentiation in the embryo, and its expression persists in adult testes. Therefore, ETV2 may serve a role in the development of male gonads and their function in adults.

At present, very little is known about how ETV2 is regulated at the molecular level. For instance, only a few interaction partners of ETV2 have been identified. These include ovo like zinc finger 2, a zinc-finger transcription factor that cooperates with ETV2 in the regulation of the FLK1 gene promoter (12), and Jumonji domain-containing (JMJD)1A, a cofactor that may repress the ETV2-mediated stimulation of matrix metalloproteinase (MMP) 1 transcription (13). Notably, JMJD1A belongs to the JMJD protein family; most members of this family have been demonstrated to be capable of demethylating histone lysine residues $(14,15)$. The present study explored whether other members of the JMJD protein family may modulate ETV2 function, and how these functions may be associated with cancer.

\section{Materials and methods}

Bioinformatics. Data regarding the mRNA expression levels of ETV2 in human tissues were obtained from the Genotype-Tissue Expression project through the Human Protein Atlas (16), accessible from www.proteinatlas.org. ETV2 gene expression and amplification in various cancers was identified with cbioportal (www.cbioportal.org).

Coimmunoprecipitation experiments. 293T cells (CRL-3216; American Type Culture Collection, Manassas, VA, USA) were grown in poly-L-lysine-coated $6-\mathrm{cm}$ plates to $\sim 25 \%$ confluency in Dulbecco's modified Eagle's medium (10-013-CV; Mediatech; Corning Inc, Corning, NY, USA) supplemented with 10\% fetal bovine serum (S11150; Atlanta Biologicals, Flowery Branch, GA, USA), as previously described (17). Subsequently, cells were transfected by the calcium phosphate coprecipitation method $(18,19)$. The following amounts of DNA were used for transfection: $1 \mu \mathrm{g}$ 6Myc-ETV2 expression construct, as previously described $(9,13)$, or $\mathrm{pCS}^{+}-6 \mathrm{Myc}$ empty vector; $2 \mu \mathrm{g}$ Flag-tagged expression plasmids encoding various JMJD proteins, or empty vector pEV3S; $6 \mu \mathrm{g}$ pBluescript $\mathrm{KS}^{+}$. At $10 \mathrm{~h}$, the precipitate was removed by 2 washes in $2 \mathrm{ml} \mathrm{PBS}$ and cells were incubated for $36 \mathrm{~h}$ in $4 \mathrm{ml}$ growth medium (20). Cells were then lysed in $675 \mu \mathrm{l}$ of $50 \mathrm{mM}$ Tris- $\mathrm{HCl}$ (pH 7.4), $50 \mathrm{mM} \mathrm{NaF}, 150 \mathrm{mM} \mathrm{NaCl}, 0.5 \%$ Igepal CA-630, $0.1 \mathrm{mM} \mathrm{Na} \mathrm{VO}_{4}, 2 \mu \mathrm{g} / \mathrm{ml}$ aprotinin, $10 \mu \mathrm{g} / \mathrm{ml}$ leupeptin, $1 \mu \mathrm{g} / \mathrm{ml}$ pepstatin $\mathrm{A}, 1 \mathrm{mM}$ phenylmethylsulfonyl fluoride, $0.1 \mathrm{mM}$ dithiothreitol and immunoprecipitations performed with anti-Flag M2 (cat. no. F1804; Sigma-Aldrich; Merck KGaA, Darmstadt, Germany) or anti-Myc 9E10 (cat. no. M4439; Sigma-Aldrich; Merck KGaA) mouse monoclonal antibodies, as previously described (21). Immunoprecipitates were subjected to SDS-PAGE; proteins were transferred onto a polyvinylidene fluoride membrane, as previously described (22). Proteins on such membranes were detected by incubation with the indicated primary antibodies followed by incubation with goat anti-mouse (cat. no. 1706516; Bio-Rad Laboratories, Inc., Hercules, CA, USA) or goat anti-rabbit (cat. no. 1706515; Bio-Rad Laboratories, Inc.) polyclonal secondary antibodies coupled to horseradish-peroxidase and visualized utilizing enhanced chemiluminescence, as previously described (23).

Reporter gene experiments. Human LNCaP prostate cancer cells (CRL-1740; American Type Culture Collection) were grown in poly-L-lysine-coated 6 -wells to $\sim 30 \%$ confluency (24) and transfected with $1 \mu \mathrm{g}$ pGL2-MMP1 (25) or pGL2-MMP7 (26) luciferase reporter plasmid, $1 \mu \mathrm{g}$ pBluescript $\mathrm{KS}^{+}$, pcDNA-ETV2 (9) or empty vector pcDNA3, and $100 \mathrm{ng}$ Flag-tagged JMJD2A or JMJD2D expression vector (27) or empty vector $\mathrm{pEV} 3 \mathrm{~S}$, with $8 \mu \mathrm{g}$ polyethylenimine. At $8-10 \mathrm{~h}$, cells were washed once with $2 \mathrm{ml}$ PBS and then incubated for another $40 \mathrm{~h}$ prior to lysis with $350 \mu \mathrm{l}$ of $25 \mathrm{mM}$ Tris, $2 \mathrm{mM}$ EDTA (pH 7.8), 1\% Triton X-100, $10 \%$ glycerol and $2 \mathrm{mM}$ dithiothreitol, as previously described (28). Luciferase activities in $100 \mu$ l lysate were determined using a luminometer $\sim 25$ min after lysis, as previously described $(29,30)$.

Statistical analysis. For luciferase reporter gene assays, the means of independent replicates with standard deviations are presented. Statistical significance was evaluated with a one-way analysis of variance followed by Tukey's multiple comparison test, and corresponding P-values were calculated with GraphPad Prism 6.0 h software (GraphPad Software, Inc., La Jolla, CA, USA). For Pearson correlations, statistical significance was estimated using a two-tailed Student's t-test. $\mathrm{P}<0.05$ was considered to indicate a statistically significant difference.

\section{Results}

Expression levels of ETV2 in normal tissue and cancer. Previously, it was reported that ETV2 expression levels in adult mouse tissues, apart from the testes, were undetectable by northern blotting $(1,9)$; however, analysis was not previously conducted in human tissue. In the present study, downloaded RNA sequencing datasets were employed to analyze ETV2 expression via bioinformatics analyses, as RNA sequencing is more sensitive than northern blotting. As expected, it was revealed that the highest levels of human ETV2 mRNA expression were observed in the testes; however, significant levels of ETV2 mRNA were also detected in a variety of other human tissues (Fig. 1A), suggesting that ETV2 may function in multiple organs in adult humans.

Similarly, the ETV2 expression levels in datasets from numerous types of human cancer from the provisional Cancer Genome Atlas (TCGA) were characterized. As presented in Fig. 1B, numerous cancer types, including uveal melanoma, pheochromocytoma and paraganglioma, or adrenocortical carcinoma displayed higher median ETV2 mRNA levels compared with other types of cancer, including acute myeloid leukemia and glioma. Notably, the high expression level of ETV2 in many types of cancer was associated with its high expression in normal tissue of that type, e.g., adrenocortical carcinoma with the adrenal gland, or hepatocellular carcinoma with the liver (compare Fig. 1B to A). In addition, the analysis of the 

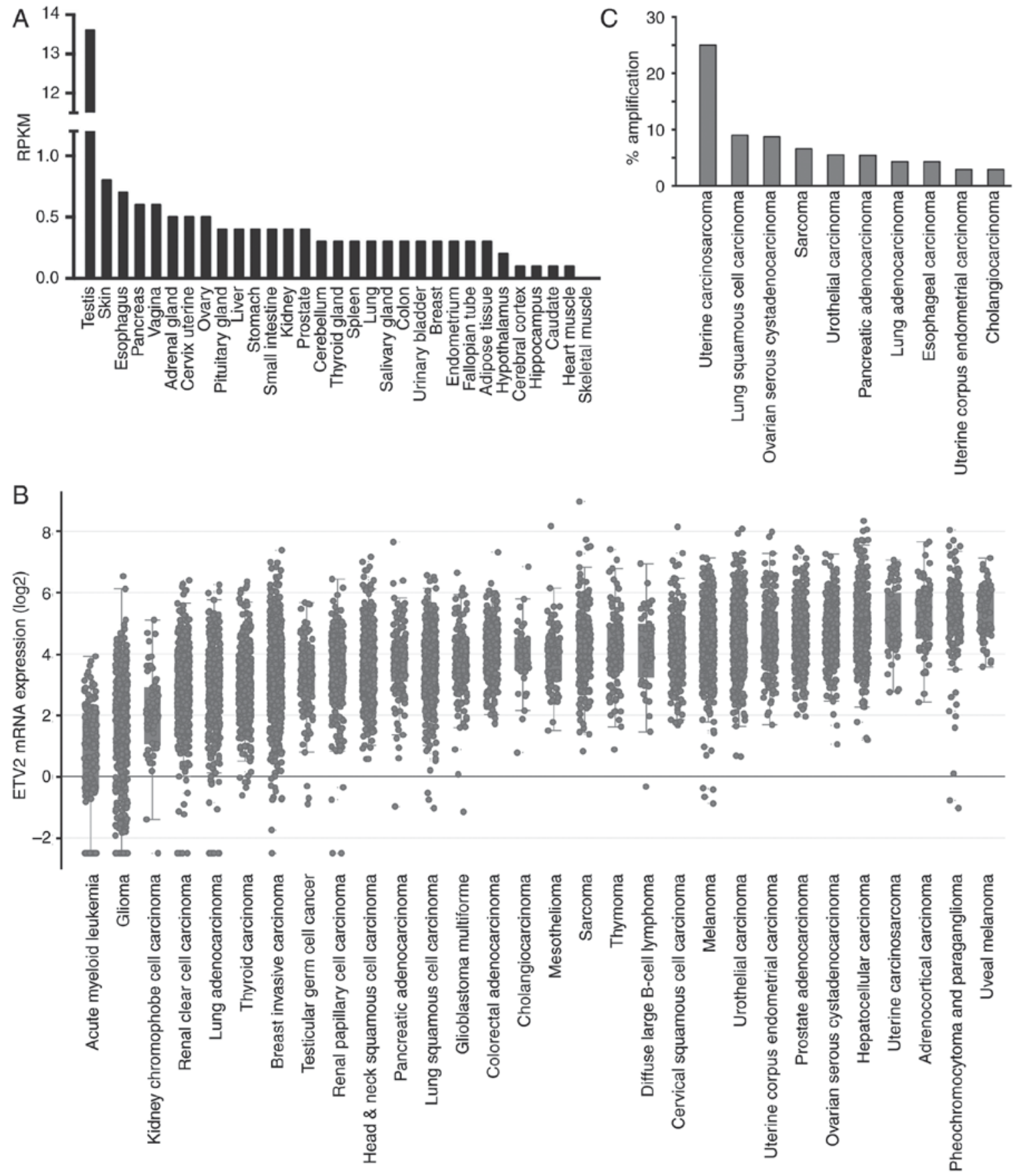

Figure 1. Expression of ETV2 in normal and cancerous tissues. (A) Expression levels of ETV2 mRNA in various human tissues. Median RPKM values from RNA sequencing studies within the Human Protein Atlas are presented. (B) ETV2 mRNA expression levels as determined by RNA sequencing in the provisional TCGA. Cancers were characterized by increasing median ETV2 mRNA expression levels. (C) Top ten highest incidences of ETV2 gene amplification presented in the provisional TCGA. ETV2, ETS variant 2; RPKM, reads per kilobase per million mapped reads; TCGA, The Cancer Genome Atlas.

provisional TCGA revealed that the ETV2 gene is amplified in various types of cancer, with the highest amplification rate $(25 \%)$ in uterine carcinosarcoma (Fig. 1C). Collectively, these data suggest that ETV2 potentially contributes to tumorigenesis.

Our laboratory is focused on prostate cancer research; it was identified that ETV2 was robustly expressed in normal prostate tissue, as well as prostate adenocarcinomas (Fig. 1A and B). In a study by Beltran et al (31) where datasets other than the provisional TCGA were used, neuroendocrine prostate cancer exhibited an ETV2 gene amplification rate of $17.8 \%$, indicating that ETV2 may be involved in prostate cancer. Previous studies have demonstrated that several JMJD2 histone demethylases, also known as lysine demethylase 4 (KDM4) proteins, are overexpressed in prostate cancer $(32,33)$. Furthermore, JMJD2A was reported to exert oncogenic functions in the prostate (33-35). Therefore, the association between ETV2, JMJD2A/KDM4A and the homolog JMJD2D/KDM4D was investigated in the present study. JMJD2B/KDM4B and JMJD2C/KDM4C were excluded from the analysis, as these proteins are highly homologous to JMJD2A/KDM4A and the scope of the study was limited by resource restraints.

Binding of ETV2 to JMJD2A and JMJD2D. To assess whether ETV2 interacts with JMJD2 proteins, Myc-tagged ETV2 

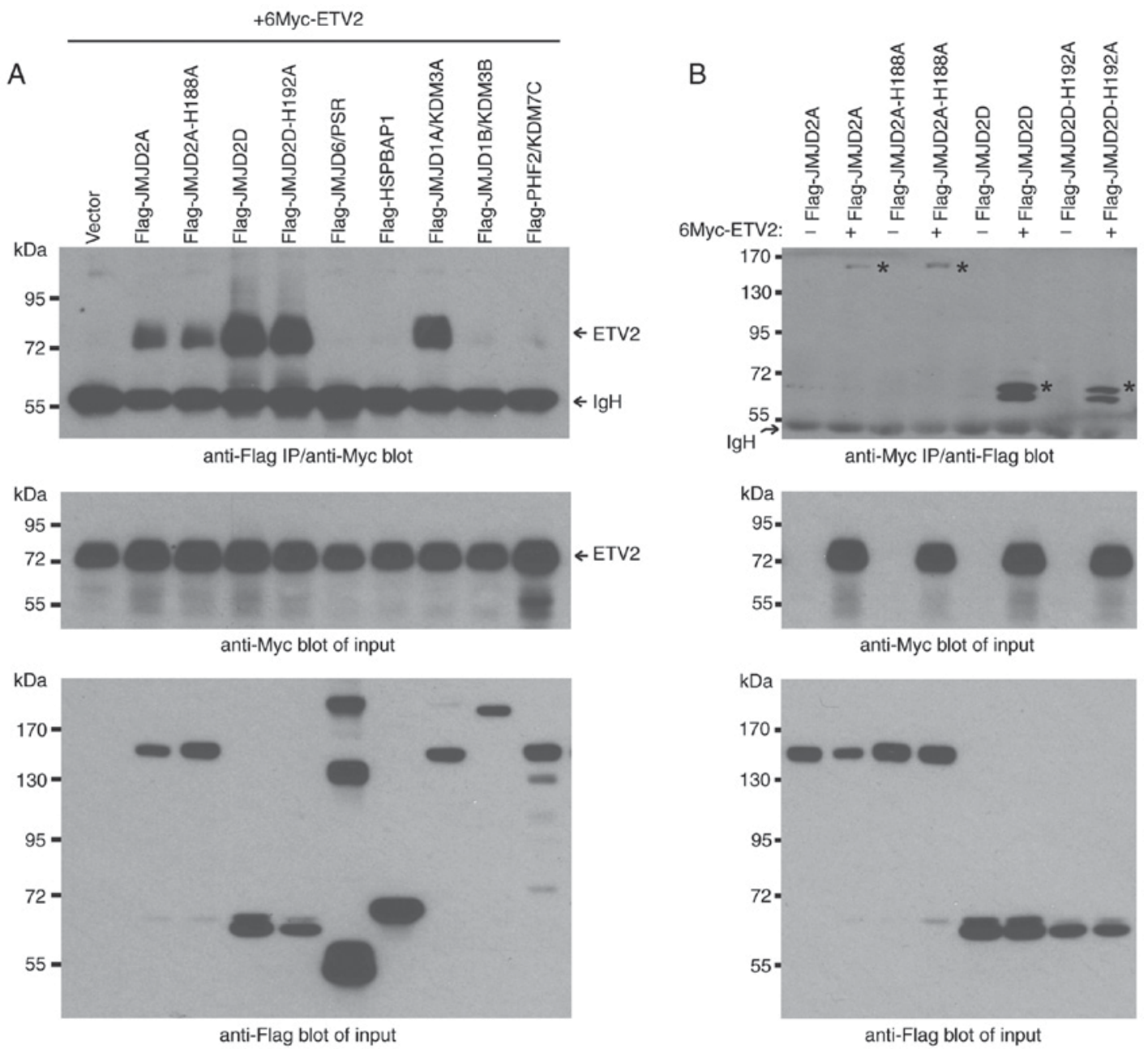

Figure 2. Interaction between ETV2 and JMJD2 proteins. (A) 6Myc-tagged ETV2 was coexpressed with indicated Flag-tagged human JMJD proteins in 293T cells. Subsequent to anti-Flag IP, coprecipitated ETV2 was detected with anti-Myc antibodies (top panel); the two bottom panels demonstrated the input levels of Myc-tagged ETV2 or Flag-tagged JMJD proteins. (B) Analogous, reverse order coimmunoprecipitation assay: Anti-Myc IP followed by anti-Flag western blotting. ETV2, ETS variant 2; JMJD, Jumonji domain-containing; IP, immunoprecipitation; IgH, immunoglobulin heavy chain.

and Flag-tagged JMJD2A or JMJD2D were coexpressed within 293 T cells. Following immunoprecipitation with anti-Flag antibodies, any coprecipitated ETV2 was detected by anti-Myc western blotting. It was revealed that ETV2 coimmunoprecipitated with JMJD2A and JMJD2D (Fig. 2A, top panel). Notably, an increased amount of ETV2 was coimmunoprecipitated with JMJD2D compared with JMJD2A, despite similar expression levels of the JMJD2 proteins and indistinguishable ETV2 expression levels (Fig. 2A, bottom panels). This indicated that ETV2 may interact more strongly with JMJD2D than JMJD2A.

As a positive control, JMJD1A (also known as KDM3A) was employed; it has previously been demonstrated to interact with ETV2 (13). This interaction was confirmed in the present study (Fig. 2A). However, ETV2 did not coimmunoprecipitate with several other JMJD proteins (including JMJD6/PSR, HSPBAP1, JMJD1B/KDM3B and PHF2/KDM7C), which indicated that ETV2 does not universally interact with JMJD proteins. Additionally, the catalytically inactive mutants JMJD2A-H188A and JMJD2D-H192A, as previously described $(36,37)$, were employed. These inactive mutants had an equivalent likelihood of coimmunoprecipitation as their wild-type counterparts, indicating that the catalytic activity of JMJD2A and JMJD2D may not be required for ETV2 complex formation (Fig. 2A).

To corroborate these results, reverse order coimmunoprecipitation experiments were conducted by the pull-down of Myc-tagged ETV2 and subsequent probing for coprecipitated Flag-tagged JMJD2 proteins (Fig. 2B). The interaction of ETV2 with JMJD2A and JMJD2D was confirmed; once again, ETV2 complexes with JMJD2D were more readily formed compared with JMJD2A, and the catalytic activity of the JMJD2 proteins was dispensable for their interaction with ETV2. JMJD2D presented as a doublet in the western blot images of Fig. 2; the higher molecular weight form appeared to be enriched in the ETV2 immunoprecipitates (compare Fig. 2B, top to bottom blot), suggesting that the higher molecular weight form of JMJD2D bound more readily to ETV2 than the lower molecular weight form. It has not been determined why a doublet was observed in the present study, or why its degree of appearance was variable from experiment to experiment. However, we hypothesize that this was due to the posttranslational modification of JMJD2D, the degree of which could be affected by the variable density of the 293T cells, their passage number and the lot of serum that was utilized to cultivate the cells. Altogether, the data presented 
in Fig. 2 indicate that ETV2 can form complexes with JMJD2A and JMJD2D in vivo.

Identification of interaction domains. To identify which region(s) of ETV2 may be required for the interaction with JMJD2 proteins, various ETV2 fragments (Fig. 3A) were coexpressed with JMJD2A or JMJD2D, and their interactions were assessed by coimmunoprecipitation. Similar to full-length ETV2 (amino acids 2-358), amino acids 23-358 interacted with JMJD2A and JMJD2D (Fig. 3B). However, further truncating ETV2 from the N-terminus down to amino acids 230-358 led to a marked reduction in the interaction with JMJD2D, whereas the interaction with JMJD2A was only slightly affected. Deletion of the $21 \mathrm{C}$-terminal amino acids from EVT2 did not affect the interaction with JMJD2A and JMJD2D (Fig. 3B; residues 23-358 and 23-337). However, a deletion of further C-terminal amino acids, including the ETS DNA-binding domain, severely compromised the ability of ETV2 to interact with JMJD2A and JMJD2D; only a low level of interaction was observable with the ETV2 amino acids 23-228, whereas amino acids 23-179 were essentially unable to form complexes with JMJD2A and JMJD2D (Fig. 3B). Collectively, this data indicated that the ETV2 amino acids 230-337 primarily mediated the interaction with JMJD2A; however, further amino acids in the 23-229 region were required for a strong interaction, in particular with JMJD2D.

Conversely, the regions within JMJD2 proteins that mediate the interaction with ETV2 were investigated by sectioning JMJD2A into three parts, and ETV2's interaction with these sections was assessed. The N-terminal JMJD2A amino acids 2-350, which encompass the conserved JmjN and JmjC domains (Fig. 4A), did not interact with ETV2 (Fig. 4B), whereas amino acids 301-703 and 704-1,064 did (Fig. 4B). The latter region encompasses two protein-protein interaction motifs, the double PHD and Tudor domains (Fig. 4A). These data indicate that two regions within JMJD2A contributed to its interaction with ETV2. The input levels for JMJD2A amino acids 704-1,064 were the lowest, but the amount of immunoprecipitated ETV2 was higher than with full-length JMJD2A (amino acids 2-1,064; Fig. 4B); therefore, the amino acids at the N-terminal from 704-1,064 may negatively affect the ability to bind to ETV2. Similar to JMJD2A, the 2-354 $\mathrm{N}$-terminal amino acids of JMJD2D, which contain the catalytic domains (Fig. 4C), did not bind to ETV2, whereas amino acids 241-523 did (Fig. 4D). In addition, the interaction of amino acids 241-523 with ETV2 appeared to be stronger compared with full-length JMJD2D, suggesting that the N-terminus of JMJD2D may exert a negative regulatory effect on the binding to ETV2. Collectively, these data reveal that the JmjN and JmjC domains of JMJD2A and JMJD2D may not be required for complex formation with ETV2. This may explain why ETV2 does not universally interact with all members of the JMJD protein family, which is characterized by the conserved JmjC domain.

Activation of MMP promoters by ETV2. ETV2 was previously reported to bind to the human MMP1 gene promoter and stimulate its activity (2). MMP7 is another MMP reported to be regulated by multiple ETS proteins, but not ETV2 (38). In addition, analysis of the MMP7 promoter for the presence of
A

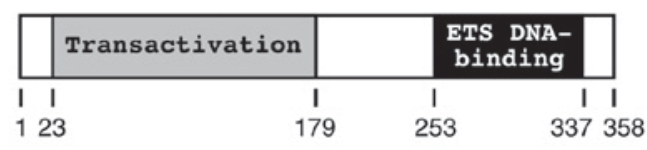

B

B 2-358 23-358 230-358 $\underline{23-179} \underline{23-228} \underline{23-337}$

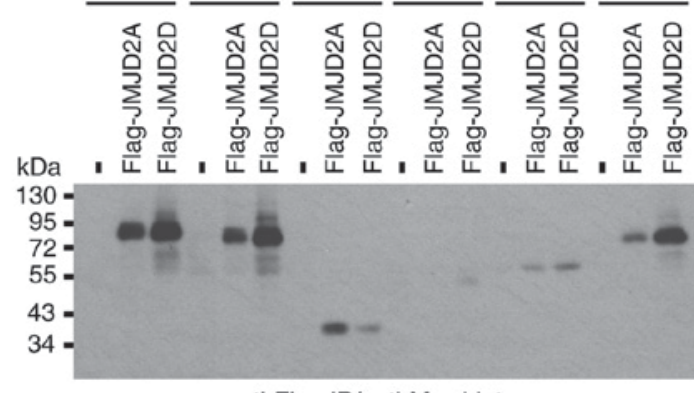

anti-Flag IP/anti-Myc blot
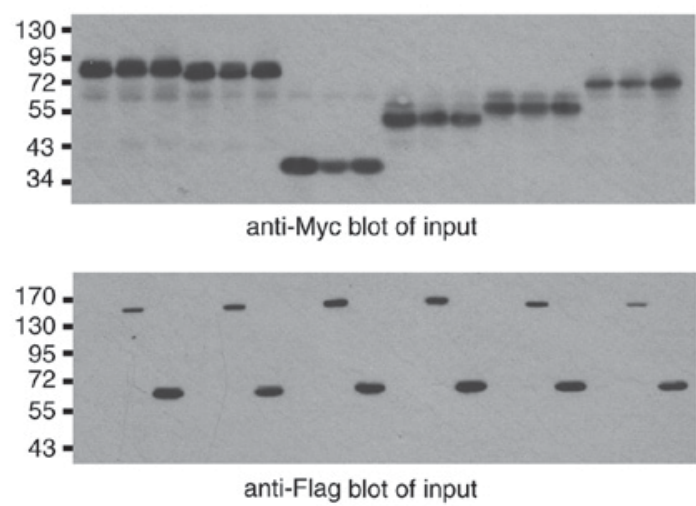

Figure 3. Identification of the JMJD2-interacting domain in ETV2. (A) Diagram of ETV2 outlining the N-terminal transactivation and the C-terminal ETS DNA-binding domains. (B) Indicated 6Myc-tagged ETV2 amino acids were coexpressed with Flag-tagged JMJD2A or JMJD2D in 293 T cells. Subsequent to anti-Flag IP, coprecipitated ETV2 fragments were revealed by anti-Myc western blotting (top panel); input levels of Myc- and flag-tagged proteins (bottom panels). JMJD, Jumonji domain-containing; ETV2, ETS variant 2; IP, immunoprecipitation.

consensus binding sites for human ETV2, [(A/G)(C/G)(C/A) $\operatorname{GGA}(\mathrm{A} / \mathrm{T})(\mathrm{G} / \mathrm{A})(\mathrm{T} / \mathrm{C})]$ (39), revealed a match that was similar to the reported binding site for ETV2 in the MMP1 promoter (Fig. 5A). Therefore, the regulation of MMP1 and MMP7 transcriptional activity by ETV2 in human $\mathrm{LNCaP}$ prostate cancer cells was investigated. ETV2 markedly stimulated the expression of an MMP1 luciferase reporter construct (Fig. 5B). Additionally, mutation of the known ETV2 binding site of the MMP1 reporter markedly reduced the ETV2-dependent transcription, but not basal transcription. The residual induction of this mutated MMP1 promoter by ETV2 may be due to further potential ETS binding sites in the MMP1 luciferase reporter construct (2), which may interact with ETV2. Regardless, these findings demonstrated that the -91/-83 ETS binding site in the MMP1 gene promoter predominantly mediated its response to ETV2 in LNCaP prostate cancer cells.

Analysis of the MMP7 gene promoter in LNCaP cells revealed reduced activity compared with the MMP1 gene promoter (Fig. 5B). However, a marked induction in luciferase activity was nonetheless observed upon the overexpression of ETV2. Additionally, mutations in the -170/-162 ETS site of the MMP7 promoter significantly decreased transactivation by ETV2 (Fig. 5B). Therefore, it was concluded that MMP1 and 
A

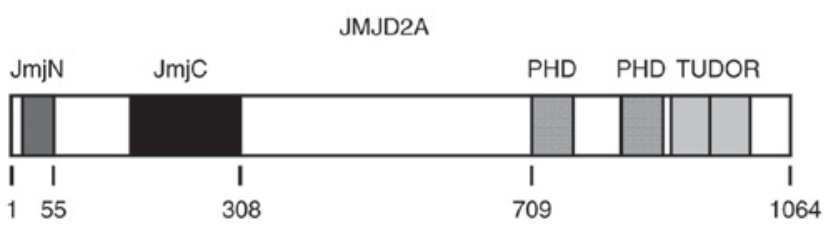

C

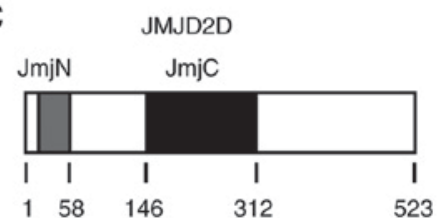

B

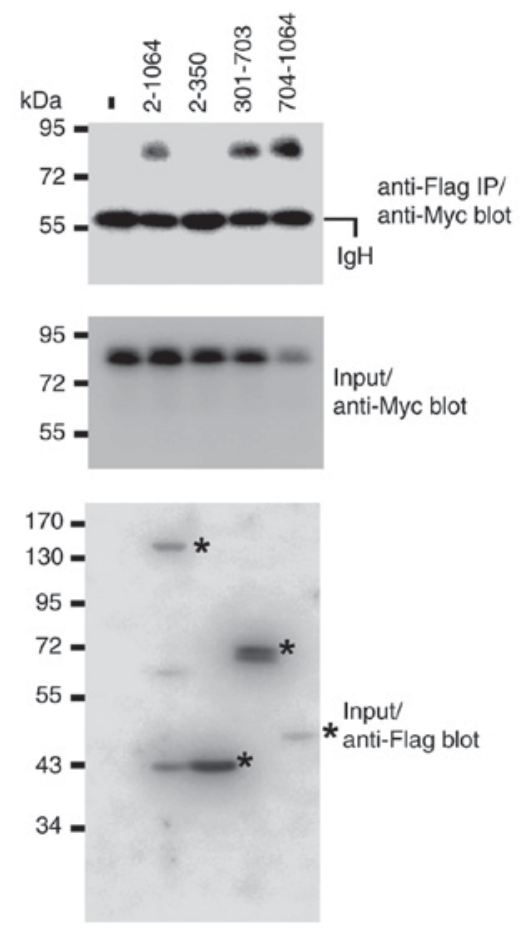

D

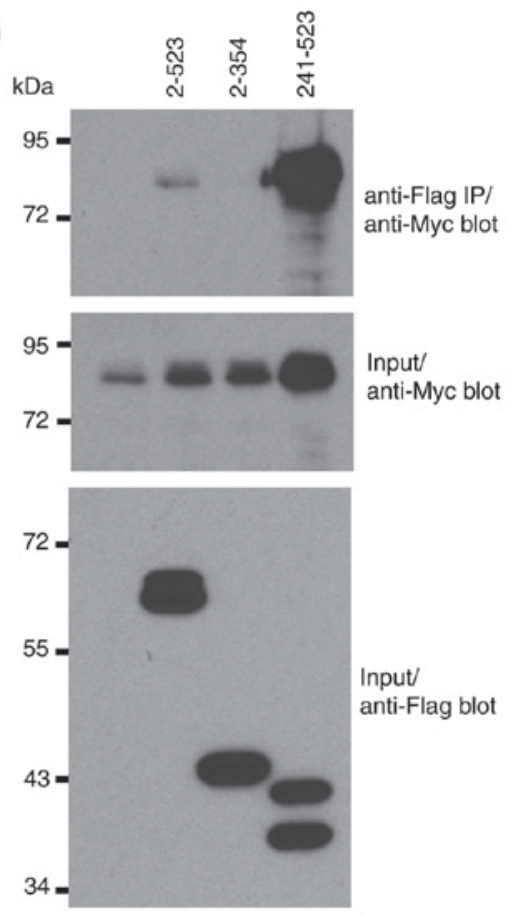

Figure 4. Determination of JMJD2 amino acids mediating complex formation with ETV2. (A) Domain structure of human JMJD2A; JmjC catalytic center is modulated by the JmjN domain. The double PHD and TUDOR domains that may bind to methylated histone lysine residues are also presented. (B) Flag-tagged JMJD2A, or indicated truncations thereof, were cotransfected with 6Myc-ETV2 into 293T cells, anti-Flag IPs performed and coprecipitated ETV2 was detected by anti-Myc western blotting (top panel); the input levels of 6Myc-ETV2 and Flag-tagged JMJD2A proteins are presented in the bottom two panels. (C) As (A), but for Flag-tagged JMJD2D; (D) as (B), but for Flag-tagged JMJD2D. JMJD, Jumonji domain-containing; ETV2, ETS variant 2; JmjC, catalytic Jumonji C domain; JmjN, Jumonji N-terminal domain; PHD, plant homeodomain; IP, immunoprecipitation.

A

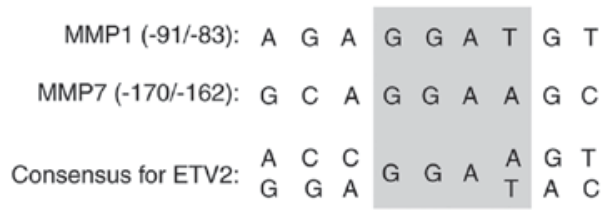

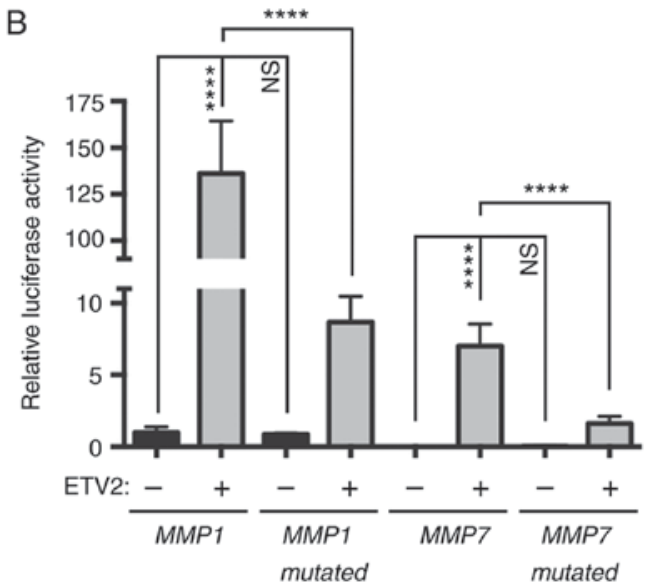

Figure 5. ETV2 stimulates the MMP1 and MMP7 promoters in LNCaP cells. (A) Comparison of ETS binding sites within the human MMP1 and MMP7 gene promoters to the consensus binding sequence for human ETV2 demonstrating the two most frequent nucleotides at each position surrounding the invariant GGA (A/T) ETS core (boxed in grey). (B) Relative luciferase activity induced by the MMP1 or MMP7 promoter in the presence or absence of 40 ng cotransfected ETV2 expression vector. The GG residues of the invariant GGA (A/T) ETS core sequence were replaced with AA in the mutated MMP1 promoter and with CC in the mutated MMP7 promoter. Statistical significance was assessed with Tukey's multiple comparisons test $(\mathrm{n}=5) .{ }^{* * * * *} \mathrm{P}<0.0001$. ETV2, ETS variant 2; MMP, matrix metalloproteinase; ETS, E26 transformation-specific; NS, not significant.

MMP7 are bona fide target genes of ETV2 in LNCaP prostate cancer cells.
Cooperation between ETV2 and JMJD2 proteins. Subsequently, the effects of ETV2 and JMJD2 coexpression on the activity of 
A

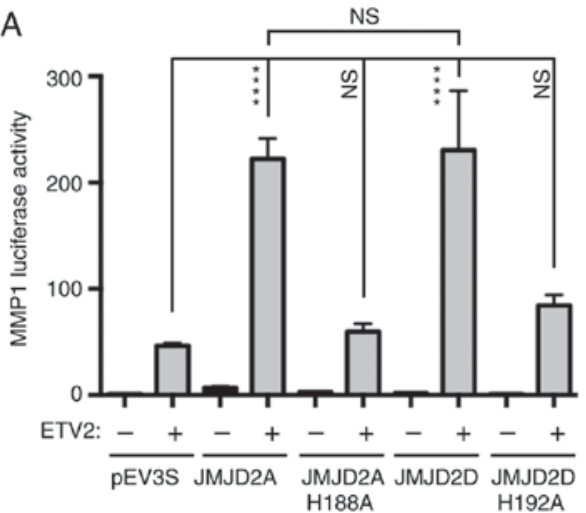

B

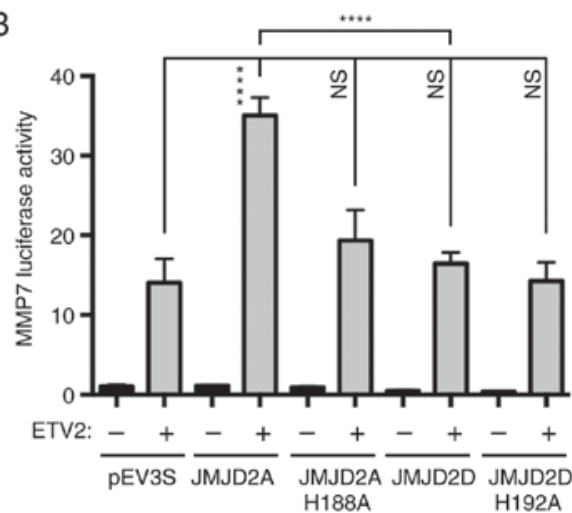

Figure 6. Functional cooperation between ETV2 and JMJD2 histone demethylases in LNCaP cells. (A) Luciferase activity induced by the activation of the MMP1 promoter by wild-type or catalytically inactive JMJD2A or JMJD2D and cotransfected ETV2 (20 ng expression plasmid); pEV3S served as the control. (B) As (A), with the MMP7 luciferase reporter gene. ${ }^{* * * *} \mathrm{P}<0.0001$, Tukey's multiple comparisons test ( $\mathrm{n}=4$ ). ETV2, ETS variant 2; JMJD, Jumonji domain-containing; MMP, matrix metalloproteinase; H188A, inactive JMJD2A mutant; H192A, inactive JMJD2D mutant; NS, not significant.
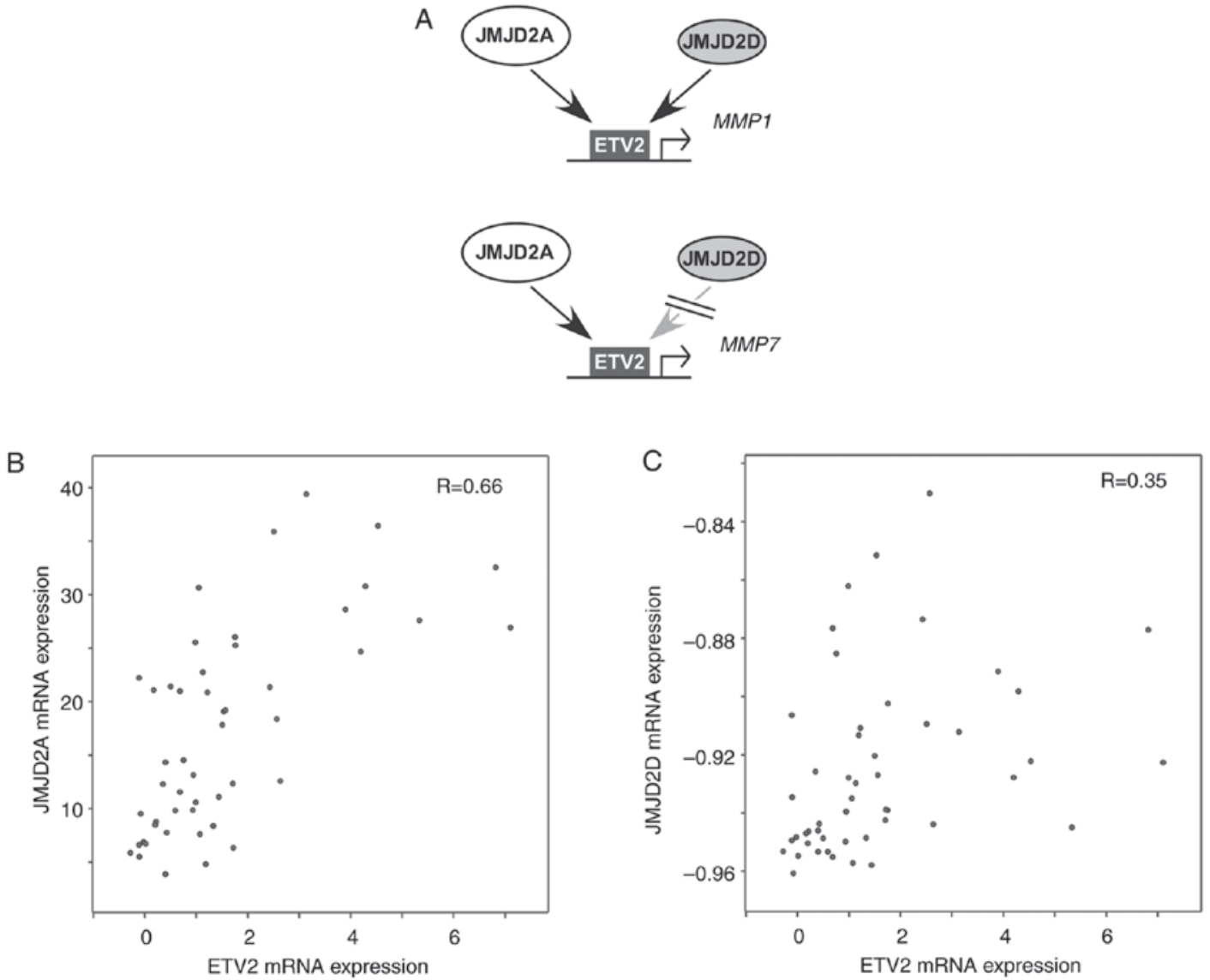

Figure 7. Functional cooperation between ETV2 and JMJD2 histone demethylases. (A) Schematic of the potential cooperation between JMJD2 proteins and ETV2. Either JMJD2A or JMJD2D activated ETV2 at the MMP1 promoter, but only JMJD2A with ETV2 activated the MMP7 promoter. JMJD2D may bind to ETV2 at the MMP7 promoter, but this is functionally neutral, or even repressive if it precludes JMJD2A from interacting with ETV2. Coexpression of ETV2 mRNA with (B) JMJD2A and (C) JMJD2D, respectively, in neuroendocrine prostate tumors (31). Both correlations were statistically significant (P<0.05). ETV2, ETS variant 2; JMJD, Jumonji domain-containing; MMP, matrix metalloproteinase.

the MMP1 gene promoter were investigated. Although ETV2 markedly induced the MMP1 luciferase activity within LNCaP cells, this did not occur when JMJD2A or JMJD2D were expressed in the absence of ETV2 (Fig. 6A). The co-expression of ETV2 with either JMJD2A or JMJD2D raised luciferase activity by $\sim 5$-fold compared with ETV2 expression alone, indicating that JMJD2A or JMJD2D synergized with ETV2 in activating transcription. Additionally, no significant difference between JMJD2A and JMJD2D was observed, despite the stronger interaction between JMJD2D and ETV2 compared with JMJD2A. In contrast to wild-type JMJD2 proteins, the catalytically inactive JMJD2A-H188A and JMJD2D-H192A mutants did not engage with ETV2 (Fig. 6A), suggesting that catalytic activity may be required for JMJD2 proteins to function as coactivators of ETV2. 
The cooperation of ETV2 with JMJD2A or JMJD2D at the MMP7 gene promoter was also investigated. As presented in Fig. 6B, JMJD2A, but not its catalytic H188A mutant, elevated ETV2-dependent transcription by 2 .5-fold, which was lower than the interaction at the MMP1 promoter. In addition, JMJD2D did not cooperate at all with ETV2 on the MMP7 promoter (Fig. 6B), indicating that JMJD2A and JMJD2D are not identical in their abilities to coactivate ETV2.

The present data suggest that ETV2 has the potential to cooperate with JMJD2 proteins to regulate transcription, as in the case of MMP1 and MMP7 genes (Fig. 7A), in LNCaP prostate cancer cells. Notably, analysis of published mRNA expression data (31) revealed that ETV2 expression was significantly correlated with expression of JMJD2A (Fig. 7B) and JMJD2D (Fig. 7C) in neuroendocrine prostate tumors, implying that the transcriptional cooperation of these factors could be relevant in this prostate cancer subtype.

\section{Discussion}

In the present study, it was identified that ETV2 forms complexes with JMJD2A or JMJD2D and that these two histone demethylases can cooperate with ETV2 to induce gene transcription within human LNCaP prostate cancer cells. JMJD2A synergized with ETV2 on the MMP1 and MMP7 promoters, whereas JMJD2D only cooperated with ETV2 on the MMP1 gene promoter (Fig. 7A). A recent study reported that JMJD2A, and not JMJD2D, may coactivate ETV1-dependent YAP1 gene transcription (33); however, both JMJD2A and JMJD2D coactivated the androgen receptor on the prostate-specific antigen and the mouse mammary tumor virus promoter (40). JMJD2A and JMJD2D may even have antagonistic activities, as exemplified by their opposing functions in p53-dependent p21 promoter regulation $(41,42)$. Thus, it would be expected in genome-wide studies for JMJD2A and JMJD2D to display different activities at a significant proportion of ETV2 target gene promoters. In addition, the ratio of JMJD2A to JMJD2D may determine the extent to which a target gene will be regulated by ETV2.

The JMJD2 gene family of histone demethylases encompasses six members in humans (43-45), suggesting that family members other than JMJD2A and JMJD2D may also cooperate with ETV2. A comparison between JMJD2A and JMJD2D revealed that they target a different spectrum of substrates: For instance, JMJD2A may target methylated lysines 9 and 36 on histone $\mathrm{H} 3$, whereas JMJD2D can only catalyze the demethylation of histone H3 lysine $9(36,46)$. This may be one reason why JMJD2A and JMJD2D behaved differently at the MMP7 gene promoter. Notably, trimethylation of histone $\mathrm{H} 3$ on lysine 9 represses the initiation of gene transcription (47), providing one explanation why demethylation of this histone mark by JMJD2A and JMJD2D may upregulate gene promoters upon recruitment by ETV2. In addition, JMJD2A contains double PHD and Tudor domains, but JMJD2D lacks any of these domains involved in binding to histone modifications, which may explain the different behaviors of JMJD2A and JMJD2D at the MMP7 promoter. For instance, the Tudor domain of JMJD2A can bind trimethylated lysine 4 on histone H3 $(48,49)$, which may protect this activating histone mark from demethylation and thereby contribute to the coactivator function of JMJD2A.
Another important finding presented in this report is that ETV2 is robustly expressed, or its gene is amplified, in various types of cancer. This suggests that ETV2 may serve a role in cancer. As MMPs are important modulators of tumor formation, invasion, angiogenesis and metastasis $(50,51)$, the activation of MMP1 and MMP7 transcription by ETV2 may contribute to its tumor-promoting function. Notably, ETV2 gene amplification was observed in lung squamous cell carcinomas and adenocarcinomas, and ETV2 expression was evident in prostate, breast and lung cancer, the three carcinomas most frequently diagnosed in humans in the western hemisphere (52). As JMJD2A has been reported to be overexpressed in prostate $(32,33)$, breast (53-56) and lung $(57,58)$ tumors, JMJD2A and ETV2 may cooperate in such tumors. Analysis of neuroendocrine prostate tumors revealed that the ETV2 gene amplification rate was $17.8 \%$ (31); in the present study, ETV2 expression was significantly correlated with JMJD2A, as well as JMJD2D. These data supported the notion that the ETV2-JMJD2A/D interaction may serve an important role in neuroendocrine prostate tumors.

In hematopoietic stem cells, ETV2 is capable of promoting their regeneration by stimulating cell proliferation $(8,59)$. In addition, JMJD2A activity appears to promote embryonic stem cell self-renewal (60). Further investigation is required to understand whether ETV2 in cooperation with JMJD2A/D may be involved in cancer stem cell maintenance and therefore, contribute to tumorigenesis. In addition, ETV2 has been identified as a reprogramming factor, either working with other factors (61-64) or alone (65-67), in converting amniotic cells or fibroblasts into endothelial cells. As cell reprogramming entails epigenetic alterations, the interactions of ETV2 with the epigenetic modifiers JMJD2A and JMJD2D, or the previously identified interacting JMJD1A (13), may be required for the generation of endothelial cells from other differentiated cells. Finally, JMJD2D is highly expressed in adult testes and regulates the methylation status of lysine 9 on histone $\mathrm{H} 3$ during spermatogenesis (68). As ETV2 is also preferentially expressed in testes $(1,9)$, the ETV2-JMJD2D complex may plausibly contribute to testicular function; however, further investigation is required.

\section{Acknowledgements}

The present study was supported by grants from the National Cancer Institute (to RJ; grant no. R01 CA154745) and funding from the Graduate School of Jilin University and the China-Japan Union Hospital of Jilin University (to XL). The content is solely the responsibility of the authors and does not necessarily represent the official views of the granting agencies.

\section{References}

1. Brown TA and McKnight SL: Specificities of protein-protein and protein-DNA interaction of GABP alpha and two newly defined ets-related proteins. Genes Dev 6: 2502-2512, 1992.

2. De Haro L and Janknecht R: Functional analysis of the transcription factor ER71 and its activation of the matrix metalloproteinase-1 promoter. Nucleic Acids Res 30: 2972-2979, 2002.

3. Hollenhorst PC, McIntosh LP and Graves BJ: Genomic and biochemical insights into the specificity of ETS transcription factors. Annu Rev Biochem 80: 437-471, 2011. 
4. Lee D, Park C, Lee H, Lugus JJ, Kim SH, Arentson E, Chung YS, Gomez G, Kyba M, Lin S, et al: ER71 acts downstream of BMP, Notch, and Wnt signaling in blood and vessel progenitor specification. Cell Stem Cell 2: 497-507, 2008.

5. Ferdous A, Caprioli A, Iacovino M, Martin CM, Morris J, Richardson JA, Latif S, Hammer RE, Harvey RP, Olson EN, et al: Nkx2-5 transactivates the Ets-related protein 71 gene and specifies an endothelial/endocardial fate in the developing embryo. Proc Natl Acad Sci USA 106: 814-819, 2009.

6. Shalaby F, Rossant J, Yamaguchi TP, Gertsenstein M, Wu XF, Breitman ML and Schuh AC: Failure of blood-island formation and vasculogenesis in Flk-1-deficient mice. Nature 376: 62-66, 1995.

7. Park C, Lee TJ, Bhang SH, Liu F, Nakamura R, Oladipupo SS, Pitha-Rowe I, Capoccia B, Choi HS, Kim TM, et al: Injury-mediated vascular regeneration requires endothelial ER71/ETV2. Arterioscler Thromb Vasc Biol 36: 86-96, 2016.

8. Lee D, Kim T and Lim DS: The Er71 is an important regulator of hematopoietic stem cells in adult mice. Stem Cells 29: 539-548, 2011.

9. De Haro L and Janknecht R: Cloning of the murine ER71 gene (Etsrp71) and initial characterization of its promoter. Genomics 85: 493-502, 2005.

10. DiTacchio L, Bowles J, Shin S, Lim DS, Koopman P and Janknecht R: Transcription factors ER71/ETV2 and SOX9 participate in a positive feedback loop in fetal and adult mouse testis. J Biol Chem 287: 23657-23666, 2012.

11. Otake T and Kuroiwa A: Molecular mechanism of male differentiation is conserved in the SRY-absent mammal, Tokudaia osimensis. Sci Rep 6: 32874, 2016.

12. Kim JY, Lee RH, Kim TM, Kim DW, Jeon YJ, Huh SH, Oh SY, Kyba M, Kataoka H, Choi K, et al: OVOL2 is a critical regulator of ER71/ETV2 in generating FLK1 ${ }^{+}$, hematopoietic, and endothelial cells from embryonic stem cells. Blood 124: 2948-2952, 2014.

13. Knebel J, De Haro L and Janknecht R: Repression of transcription by TSGA/Jmjd1a, a novel interaction partner of the ETS protein ER71. J Cell Biochem 99: 319-329, 2006.

14. Yamane K, Toumazou C, Tsukada Y, Erdjument-Bromage H, Tempst P, Wong J and Zhang Y: JHDM2A, a JmjC-containing H3K9 demethylase, facilitates transcription activation by androgen receptor. Cell 125: 483-495, 2006.

15. Kooistra SM and Helin K: Molecular mechanisms and potential functions of histone demethylases. Nat Rev Mol Cell Biol 13: 297-311, 2012.

16. Uhlén M, Fagerberg L, Hallström BM, Lindskog C, Oksvold P, Mardinoglu A, Sivertsson A, Kampf C, Sjöstedt E, Asplund A, et al: Proteomics. Tissue-based map of the human proteome. Science 347: 1260419, 2015.

17. Mooney SM, Grande JP, Salisbury JL and Janknecht R Sumoylation of p68 and p72 RNA helicases affects protein stability and transactivation potential. Biochemistry 49: 1-10, 2010.

18. Dowdy SC, Mariani A and Janknecht R: HER2/Neu- and TAK1-mediated up-regulation of the transforming growth factor beta inhibitor Smad7 via the ETS protein ER81. J Biol Chem 278: 44377-44384, 2003.

19. Janknecht R: Regulation of the ER81 transcription factor and its coactivators by mitogen- and stress-activated protein kinase 1 (MSK1). Oncogene 22: 746-755, 2003.

20. Kim TD, Fuchs JR, Schwartz E, Abdelhamid D, Etter J, Berry WL, Li C, Ihnat MA, Li PK and Janknecht R: Pro-growth role of the JMJD2C histone demethylase in HCT-116 colon cancer cells and identification of curcuminoids as JMJD2 inhibitors. Am J Transl Res 6: 236-247, 2014

21. Berry WL, Kim TD and Janknecht R: Stimulation of $\beta$-catenin and colon cancer cell growth by the KDM4B histone demethylase. Int J Oncol 44: 1341-1348, 2014.

22. Papoutsopoulou S and Janknecht R: Phosphorylation of ETS transcription factor ER81 in a complex with its coactivator CREB-binding protein and p300. Mol Cell Biol 20: 7300-7310, 2000.

23. Shin S, Kim TD, Jin F, van Deursen JM, Dehm SM, Tindall DJ, Grande JP, Munz JM, Vasmatzis G and Janknecht R: Induction of prostatic intraepithelial neoplasia and modulation of androgen receptor by ETS variant 1/ETS-related protein 81 . Cancer Res 69: 8102-8110, 2009

24. Oh S, Shin S, Lightfoot SA and Janknecht R: 14-3-3 proteins modulate the ETS transcription factor ETV1 in prostate cancer. Cancer Res 73: 5110-5119, 2013
25. Bosc DG, Goueli BS and Janknecht R: HER2/Neu-mediated activation of the ETS transcription factor ER81 and its target gene MMP-1. Oncogene 20: 6215-6224, 2001.

26. Shin S, Oh S, An S and Janknecht R: ETS variant 1 regulates matrix metalloproteinase-7 transcription in $\mathrm{LNCaP}$ prostate cancer cells. Oncol Rep 29: 306-314, 2013.

27. Shin S and Janknecht R: Diversity within the JMJD2 histone demethylase family. Biochem Biophys Res Commun 353: 973-977, 2007.

28. Mooney SM, Goel A, D'Assoro AB, Salisbury JL and Janknecht R: Pleiotropic effects of p300-mediated acetylation on p68 and p72 RNA helicase. J Biol Chem 285: 30443-30452, 2010.

29. Wu J and Janknecht R: Regulation of the ETS transcription factor ER81 by the $90-\mathrm{kDa}$ ribosomal S6 kinase 1 and protein kinase A. J Biol Chem 277: 42669-42679, 2002.

30. Goel A and Janknecht R: Concerted activation of ETS protein ER81 by p160 coactivators, the acetyltransferase p300 and the receptor tyrosine kinase HER2/Neu. J Biol Chem 279: 14909-14916, 2004.

31. Beltran H, Prandi D, Mosquera JM, Benelli M, Puca L, Cyrta J, Marotz C, Giannopoulou E, Chakravarthi BV, Varambally S, et al: Divergent clonal evolution of castration-resistant neuroendocrine prostate cancer. Nat Med 22: 298-305, 2016

32. Cloos PA, Christensen J, Agger K, Maiolica A, Rappsilber J, Antal T, Hansen KH and Helin K: The putative oncogene GASC1 demethylates tri- and dimethylated lysine 9 on histone $\mathrm{H} 3$. Nature 442: 307-311, 2006.

33. Kim TD, Jin F, Shin S, Oh S, Lightfoot SA, Grande JP, Johnson AJ, van Deursen JM, Wren JD and Janknecht R: Histone demethylase JMJD2A drives prostate tumorigenesis through transcription factor ETV1. J Clin Invest 126: 706-720, 2016.

34. Kim TD, Shin S and Janknecht R: ETS transcription factor ERG cooperates with histone demethylase KDM4A. Oncol Rep 35: 3679-3688, 2016

35. Kim TD, Oh S, Lightfoot SA, Shin S, Wren JD and Janknecht R: Upregulation of PSMD10 caused by the JMJD2A histone demethylase. Int J Clin Exp Med 9: 10123-10134, 2016.

36. Whetstine JR, Nottke A, Lan F, Huarte M, Smolikov S, Chen Z, Spooner E, Li E, Zhang G, Colaiacovo M and Shi Y: Reversal of histone lysine trimethylation by the JMJD2 family of histone demethylases. Cell 125: 467-481, 2006.

37. Klose RJ, Yamane K, Bae Y, Zhang D, Erdjument-Bromage H, Tempst P, Wong J and Zhang Y: The transcriptional repressor JHDM3A demethylates trimethyl histone H3 lysine 9 and lysine 36. Nature 442: 312-316, 2006.

38. Crawford HC, Fingleton B, Gustavson MD, Kurpios N, Wagenaar RA,Hassell JA and Matrisian LM: The PEA3 subfamily of Ets transcription factors synergizes with beta-catenin-LEF-1 to activate matrilysin transcription in intestinal tumors. Mol Cell Biol 21: 1370-1383, 2001.

39. Wei GH, Badis G, Berger MF, Kivioja T, Palin K, Enge M, Bonke M, Jolma A, Varjosalo M, Gehrke AR, et al: Genome-wide analysis of ETS-family DNA-binding in vitro and in vivo. EMBO J 29: 2147-2160, 2010

40. Shin S and Janknecht R: Activation of androgen receptor by histone demethylases JMJD2A and JMJD2D. Biochem Biophys Res Commun 359: 742-746, 2007.

41. Kim TD, Shin S, Berry WL, Oh S and Janknecht R: The JMJD2A demethylase regulates apoptosis and proliferation in colon cancer cells. J Cell Biochem 113: 1368-1376, 2012.

42. Kim TD, Oh S, Shin S and Janknecht R: Regulation of tumor suppressor 553 and HCT116 cell physiology by histone demethylase JMJD2D/KDM4D. PLoS One 7: e34618, 2012.

43. Katoh $\mathrm{M}$ and Katoh M: Identification and characterization of JMJD2 family genes in silico. Int J Oncol 24: 1623-1628, 2004

44. Berry WL and Janknecht R: KDM4/JMJD2 histone demethylases: Epigenetic regulators in cancer cells. Cancer Res 73 2936-2942, 2013

45. Labbe RM, Holowatyj A and Yang ZQ: Histone lysine demethylase (KDM) subfamily 4: Structures, functions and therapeutic potential. Am J Transl Res 6: 1-15, 2013.

46. Hillringhaus L, Yue WW, Rose NR, Ng SS, Gileadi C, Loenarz C, Bello SH, Bray JE, Schofield CJ and Oppermann U: Structural and evolutionary basis for the dual substrate selectivity of human KDM4 histone demethylase family. J Biol Chem 286: 41616-41625, 2011

47. Black JC, Van Rechem C and Whetstine JR: Histone lysine methylation dynamics: Establishment, regulation, and biological impact. Mol Cell 48: 491-507, 2012. 
48. Kim J, Daniel J, Espejo A, Lake A, Krishna M, Xia L, Zhang Y and Bedford MT: Tudor, MBT and chromo domains gauge the degree of lysine methylation. EMBO Rep 7: 397-403, 2006.

49. Huang Y, Fang J, Bedford MT, Zhang Y and Xu RM: Recognition of histone $\mathrm{H} 3$ lysine- 4 methylation by the double tudor domain of JMJD2A. Science 312: 748-751, 2006.

50. Hadler-Olsen E, Winberg JO and Uhlin-Hansen L: Matrix metalloproteinases in cancer: Their value as diagnostic and prognostic markers and therapeutic targets. Tumour Biol 34: 2041-2051, 2013.

51. Shay G, Lynch CC and Fingleton B: Moving targets: Emerging roles for MMPs in cancer progression and metastasis. Matrix Biol 44-46: 200-206, 2015.

52. Siegel RL, Miller KD and Jemal A: Cancer statistics, 2017. CA Cancer J Clin 67: 7-30, 2017.

53. Patani N, Jiang WG, Newbold RF and Mokbel K: Histone-modifier gene expression profiles are associated with pathological and clinical outcomes in human breast cancer. Anticancer Res 31: 4115-4125, 2011.

54. Slee RB, Steiner CM,Herbert BS, Vance GH, Hickey RJ, Schwarz T, Christan S, Radovich M, Schneider BP, Schindelhauer D and Grimes BR: Cancer-associated alteration of pericentromeric heterochromatin may contribute to chromosome instability. Oncogene 31: 3244-3253, 2012.

55. Berry WL, Shin S, Lightfoot SA and Janknecht R: Oncogenic features of the JMJD2A histone demethylase in breast cancer. Int J Oncol 41: 1701-1706, 2012.

56. Li LL, Xue AM, Li BX, Shen YW, Li YH, Luo CL, Zhang MC, Jiang JQ, Xu ZD, Xie JH and Zhao ZQ: JMJD2A contributes to breast cancer progression through transcriptional repression of the tumor suppressor ARHI. Breast Cancer Res 16: R56, 2014.

57. Mallette FA and Richard S: JMJD2A promotes cellular transformation by blocking cellular senescence through transcriptional repression of the tumor suppressor CHD5. Cell Rep 2: 1233-1243, 2012.

58. Xu W, Jiang K, Shen M, Qian Y and Peng Y: SIRT2 suppresses non-small cell lung cancer growth by targeting JMJD2A. Biol Chem 396: 929-936, 2015.

59. Xu CX, Lee TJ, Sakurai N, Krchma K, Liu F, Li D, Wang T and Choi K: ETV2/ER71 regulates hematopoietic regeneration by promoting hematopoietic stem cell proliferation. J Exp Med 214: $1643-1653,2017$.
60. Pedersen MT, Kooistra SM, Radzisheuskaya A, Laugesen A, Johansen JV, Hayward DG, Nilsson J, Agger K and Helin K: Continual removal of $\mathrm{H} 3 \mathrm{~K} 9$ promoter methylation by Jmjd2 demethylases is vital for ESC self-renewal and early development. EMBO J 35: 1550-1564, 2016.

61. Ginsberg M, James D, Ding BS, Nolan D, Geng F, Butler JM, Schachterle W, Pulijaal VR, Mathew S, Chasen ST, et al: Efficient direct reprogramming of mature amniotic cells into endothelial cells by ETS factors and TGF $\beta$ suppression. Cell 151: 559-575, 2012.

62. Han JK, Chang SH, Cho HJ, Choi SB, Ahn HS, Lee J, Jeong H, Youn SW, Lee HJ, Kwon YW, et al: Direct conversion of adult skin fibroblasts to endothelial cells by defined factors. Circulation 130: 1168-1178, 2014.

63. Ginsberg M, Schachterle W, Shido K and Rafii S: Direct conversion of human amniotic cells into endothelial cells without transitioning through a pluripotent state. Nat Protoc 10: 1975-1985, 2015

64. Wong WT and Cooke JP: Therapeutic transdifferentiation of human fibroblasts into endothelial cells using forced expression of lineage-specific transcription factors. J Tissue Eng 7, 2016.

65. Morita R, Suzuki M, Kasahara H, Shimizu N, Shichita T, Sekiya T, Kimura A, Sasaki K, Yasukawa H and Yoshimura A: ETS transcription factor ETV2 directly converts human fibroblasts into functional endothelial cells. Proc Natl Acad Sci USA 112: 160-165, 2015.

66. Van Pham P, Vu NB, Nguyen HT, Huynh OT and Truong MT: Significant improvement of direct reprogramming efficacy of fibroblasts into progenitor endothelial cells by ETV2 and hypoxia. Stem Cell Res Ther 7: 104, 2016.

67. Lee S, Park C, Han JW, Kim JY, Cho K, Kim EJ, Kim S, Lee SJ, Oh SY, Tanaka Y, et al: Direct reprogramming of human dermal fibroblasts into endothelial cells using ER71/ETV2. Circ Res 120: 848-861, 2017.

68. Iwamori N, Zhao M, Meistrich ML and Matzuk MM: The testis-enriched histone demethylase, KDM4D, regulates methylation of histone $\mathrm{H} 3$ lysine 9 during spermatogenesis in the mouse but is dispensable for fertility. Biol Reprod 84: 1225-1234, 2011. 